\title{
Thermal Cis-Trans Isomerization of Solvatochromic Merocyanines: Linear Correlations between Solvent Polarity and Adlabatic and Dlabatic Transition Energies
}

\author{
Shakir T. Abdel-Halim, Mahmoud H. Abdel-Kader, ${ }^{\dagger}$ \\ Department of Chemistry, Faculty of Science, University of Tanta, Tanta, Egypt \\ and Ulrich E. Steiner* \\ Fakultät für Chemie, Universität Konstanz, D-7750 Konstanz, Federal Republic of Germany \\ (Received: January 11, 1988)
}

\begin{abstract}
The correlation between solvatochromy and the solvent-polarity-dependent rate constant $k_{\mathrm{ct}}$ of thermal cis-trans isomerization (solvatokinetic behavior) has been investigated for a stilbazolium-type merocyanine and an amphiphilic analogue in a polarity series of eight neat solvents (protic and aprotic). At room temperature $k_{\mathrm{ct}}$ varies over 7 orders of magnitude, decreasing with increasing solvent polarity. It has been demonstrated that the first transition energy $\Delta E_{\max }$ and the free activation enthalpy $\Delta G^{*}$ ct of thermal cis-trans isomerization are linearly correlated with each other and with Dimroth's solvent polarity parameter $E_{\mathrm{T}}$. These observations and the solvent-polarity-dependent increase of absorption band width are explained in terms of a model emphasizing the strong coupling between molecular conformation, electronic charge distribution, and solvent polarization.
\end{abstract}

\section{Introduction}

The solvatochromism of merocyanine dyes was first reported in 1951 by Brooker et al. ${ }^{1}$ The extraordinary sensitivity of the electronic absorption spectra of these compounds to medium effects permits their use as sensitive probes of solvent polarity, which may be applied, e.g., for spectrophotometric determination of water in organic solvents. ${ }^{2}$ They have been used as sensitizers in color photography, ${ }^{3}$ and due to their extreme hyperpolarizability, ${ }^{4,5}$ they are promising materials for nonlinear optics. ${ }^{6}$ Among this class of compounds particularly the stilbazolium betaine $M$ has continued to be the subject of extensive experimental and theoretical investigation. ${ }^{7-16}$



(q)

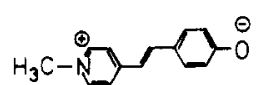

(b)
Whereas earlier solvatochromic studies had been carried out in mixed-solvent solutions, ${ }^{1,14}$ only recently a comprehensive investigation in 26 different solvents has been reported by Jacques. ${ }^{12}$ The strongly negative solvatochromy (increase of electronic transition energy $\Delta E_{\max }$ with solvent polarity) was shown to be linearly correlated with Dimroth's empirical solvent polarity parameter $E_{\mathrm{T}}$ for solvents with $E_{\mathrm{T}} \geq 36$. Moreover, a linear twoparameter correlation with Taft's $\pi^{*}$ (nonspecific solvent polarity) and $\alpha$ (hydrogen-bond-donor ability) ${ }^{17}$ has been established.

Negative solvatochromy is generally indicative of a decrease of dipole moment upon electronic excitation. However, the extremely strong effect in the case of compounds like $M$ involves an additional enhancement due to an increasing ground-state dipole moment with increasing solvent polarity. 4,10 This may be attributed to an increasing participation of the benzenoid, zwitterionic structure $M(b)$ in polar solvents, which has been borne out by several quantum chemical calculations of various levels of sophistication. ${ }^{14-16}$

One should expect that, in parallel with an increasing contribution of resonance structure $\mathrm{M}(\mathrm{b})$, an increasing double-bond character of the central $\mathrm{C}-\mathrm{C}$ bond should develop, which should be reflected in a "solvatokinetic" behavior of cis-trans isomerization around this bond. An indication of such an effect is the largely different rate of cis-trans isomerization that we reported previously for $\mathrm{M}$ and its protonated form $\mathrm{MH}^{+}$in water. ${ }^{18}$ In fact, $\mathrm{MH}^{+}$, where the oxygen atom in $\mathrm{M}$ is protonated, represents a limiting case of the $\mathrm{q} \leftrightarrow \mathrm{b}$ resonance, with a predominant $\mathrm{b}$

\footnotetext{
Present address: College of Medicine and Medical Science, Arabian Gulf
} University, PO Box 26671, Adlya, Bahrain. contribution. In a recent MNDOC calculation by Tavan and Schulten, ${ }^{16}$ who compared the isomerization potential of $M$ and $\mathrm{MH}^{+}$, this feature was clearly reproduced.

An example of a correlation between solvatochromy and solvent-dependent kinetics (solvatokinetics) has been previously reported by Whitten and collaborators ${ }^{19}$ for the solvatochromic compound 4-(diethylamino)-4'-nitroazobenzene (DENAB).

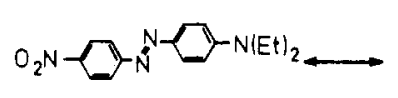

(b)<smiles>CCN(CC)C1CCC(N=NC2CCC(N=O)CC2)CC1</smiles>

(q)
Here, however, the dipolar properties of $q$ and $b$ resonance structures are opposite from the case of merocyanine $M$, and, in fact, it was observed that in polar solvents, which energetically favor the dipolar resonance form $q$ and cause a hypsochromic shift of the first absorption band, the activation free enthalpy $\Delta G^{*}$ ct has a low value.

In view of the general interest in the properties of merocyanine $M$ and to generalize the reported findings for DENAB, we investigated the relation between solvatochromic and solvatokinetic

(1) Brooker, L. G. S.; Keyes, G. H.; Heseltine, D. W. J. Am. Chem. Soc. $1951,73,5332$.

(2) Kumoi, S.; Oyama, K.; Yano, T.; Kobayashi, H.; Ueno, K. Talanta $1970,17,319$.

(3) Brooker, L. G. S.; Keyes, G. H.; Sprague, R. H.; van Dyke, R. H.; van Lare, E.; van Zandt, G.; White, F. L. J. Am. Chem. Soc. 1951, 73, 5326

(4) Levine, B. F.; Bethey, C. G.; Wassermann, E.; Leenders, L. J. Chem. Phys. 1978, 68, 5042 .

(5) Dulcic, A.; Flytzanis, C. Opt. Commun. 1978, 25, 402.

(6) Williams, D. J. Angew. Chem. 1984, 96, 637.

(7) Hünig, S.; Rosenthal, O. Liebigs Ann. Chem. 1955, 92, 161

(8) Lippert, E. Z. Elektrochem. 1957, 61, 963. Lippert, E.; Moll, F. Ibid. 1954, 58,718

(9) Bayliss, N. S.; McRae, G. J. Am. Chem. Soc. 1952, 74, 5803.

(10) Varma, C. A. G. O.; Groenen, E. J. J. Recl. Trav. Chim. Pays-Bas 1972, 91, 296

(11) Al-Hassan, K. A.; El-Bayoumi, M. A. Chem. Phys. Lett. 1980, 76, 121

(12) Jacques, P. J. Phys. Chem. 1984, 90, 5535.

(13) Rasch, G. Z. Chem. (Leipzig) 1962, 2, 5.

(14) Benson, H. G.; Murrell, J. N. J. Chem. Soc., Faraday Trans. 21972, $68,137$.

(15) Botrel, A.; Beuze, A.; Jacques, P.; Strub, H. J. Chem. Soc., Faraday Trans. 2 1984, 80, 1235.

(16) Tavan, P.; Schulten, K. Chem. Phys. Lett. 1984, 110, 191.

(17) Kamlet, M. J.; Abboud, J.-L. M.; Abraham, M. H.; Taft, R. W. J. Org. Chem. 1983, 48, 2877.

(18) Steiner, U. E.; Abdel-Kader, M. H.; Fischer, P.; Kramer, H. E. A. J. Am. Chem. Soc. 1978, 100, 3190 . 2808 . 
SCHEME I

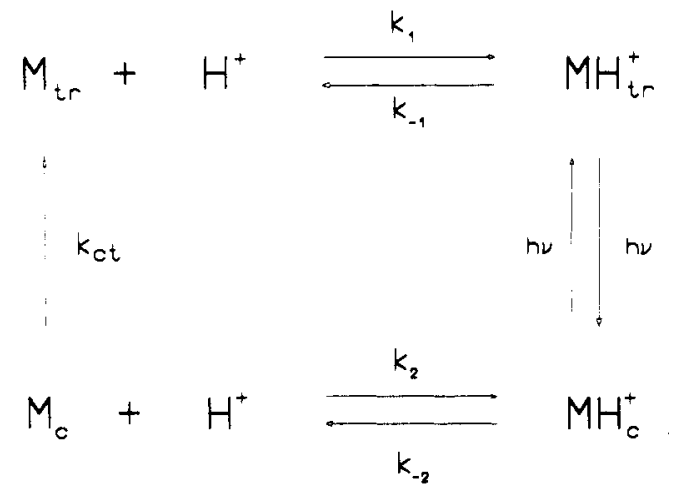

behavior for the merocyanine $\mathrm{M}$ and an amphiphilic derivative $\mathrm{C}_{16} \mathrm{M}$ (where $\mathrm{CH}_{3}$ is replaced by $\mathrm{C}_{16} \mathrm{H}_{33}$ ), which had been synthesized as a special polarity probe for micellar systems.

\section{Experimental Section}

Materials and Instrumentation. The merocyanines $\mathrm{M}$ and $\mathrm{C}_{16} \mathrm{M}$ were synthesized according to literature procedures. ${ }^{20,21}$ The methylated compound $M$ was recrystallized several times from water, the cetyl-substituted compound $\mathrm{C}_{16} \mathrm{M}$ from ethyl alcohol containing traces of ammonia. The purity of the dyes was checked by thin-layer chromatography and elemental analysis. According to the latter and to the results of $\mathrm{H}_{2} \mathrm{O}$ determination with a Mitsubishi moisturemeter the crystal water content corresponded to $3 \mathrm{H}_{2} \mathrm{O}$ for $\mathrm{M}$ and $2.5 \mathrm{H}_{2} \mathrm{O}$ for $\mathrm{C}_{16} \mathrm{M}$.

Organic solvents (Merck) were of reagent grade and used without further purification. Buffer materials used were piperidine (Merck, p.A.), 1,5-diazabicyclo[4.3.0]non-5-ene (DBU; (Merck, >97\%) $\mathrm{H}_{2} \mathrm{SO}_{4}$ (95-97\%, Merck, p.A.), $1 \mathrm{~N} \mathrm{NaOH}$ (Merck, Titrisol), and phthalic acid (Fluka, 99.5\%). Sodium methylate was prepared freshly from metallic sodium and methanol.

Absorption spectra were recorded on a Perkin-Elmer Lambda 5 spectrometer, with $1.0-\mathrm{cm}$ matched quartz cells with a cuvette holder connected to a thermostat. The slow kinetic runs in ethylene glycol and methanol were followed in the UV spectrometer after photochemical trans-cis isomerization by irradiation with light from the 365-nm line of a high-pressure $\mathrm{Hg}$ lamp (Osram HBO $200 \mathrm{~W} / 2$ ) selected by a $365-\mathrm{nm}$ interference filter. The fast kinetic runs in other organic solvents were carried out in a flash photolysis apparatus (Applied Photophysics Model KR-1), with double-wall cylindrical cuvettes of $10-\mathrm{cm}$ path length connected to a thermostat.

Method of Investigation. As reported previously ${ }^{18}$ the cis isomer of the basic merocyanine $\mathrm{M}$ cannot be obtained in a direct photochemical process, e.g., as in the case of DENAB, since the photochemical quantum yield $\Phi_{\mathrm{ct}}(\mathrm{M})$ is close to zero. On the other hand, the protonated form $\mathrm{MH}^{+}$can be efficiently photoisomerized. Thus, to measure the rate constant $k_{\mathrm{ct}}$ of the process $\mathbf{M}_{\mathrm{c}}$ $\rightarrow \mathrm{M}_{\mathrm{tr}}$, we made use of the previously investigated protolyticphotochemical-thermal reaction cycle depicted in Scheme I. If it is assumed that the protolytic equilibria are rapidly established, the mixtures $\mathrm{M}_{\mathrm{c}} / \mathrm{MH}_{\mathrm{c}}{ }^{+}$and $\mathrm{M}_{\mathrm{tr}} / \mathrm{MH}_{\mathrm{tr}}{ }^{+}$will kinetically behave as quasispecies, and their thermal turnover following the precedent obverse photochemical turnover, will be a first-order process with an effective rate constant of

$$
k_{\mathrm{eff}}=\alpha_{\mathrm{c}} k_{\mathrm{ct}}
$$

where $\alpha_{c}$ is defined as

$$
\alpha_{c}=\frac{\left[\mathrm{M}_{\mathrm{c}}\right]}{\left[\mathrm{M}_{\mathrm{c}}\right]+\left[\mathrm{MH}_{\mathrm{c}}^{+}\right]}
$$

Thus $\alpha_{\mathrm{c}}$ has to be known to determine $k_{\mathrm{ct}}$ from $k_{\text {eff }}$. Our previous investigations in aqueous solution have shown that $\alpha_{c}$

(20) Minch, M. J.; Sadig Shah, S. J. Chem. Educ. 1977, 54, 709

(21) Donchi, K. F.; Robert, G. P.; Ternai, B.; Derrick, P. J. Aust. J. Chem. $1980,33,2199$.



Figure 1. Absorption spectra of $\mathrm{C}_{16} \mathrm{M}$ in various solvents: 2, ethylene glycol; 3, methanol; 4, 2-propanol; 5, acetonitrile; $6, N, N$-dimethylformamide; 7 , dimethyl sulfoxide; 8 , acetone.

and $\alpha_{\mathrm{tr}}$ are virtually identical. We assumed this equality also to hold for other solvents. The $\alpha$ value in a given solution was determined as the ratio of the long-wavelength absorption maximum to that of a solution with the equilibrium completely on the side of the $\mathbf{M}$ form. For observation of the pure $M$ spectra, the $\mathrm{MH}^{+} / \mathrm{M}$ equilibrium had to be completely shifted to the $\mathrm{M}$ form, which was achieved by adding between $10^{-3}$ and $10^{-2} \mathrm{M}$ of piperidine or DBN in aprotic solvents or $10^{-2} \mathrm{M} \mathrm{NaOH}$ in protic solvents. To fix $\alpha_{\mathrm{tr}}$ at a value of about 0.5 in each solvent investigated, we added suitable amounts of piperidine and sulfuric acid at a total concentration on the order of $10^{-3} \mathrm{M}$ as a buffer system. In the case of methanol a standard buffer of $\mathrm{pH}^{*} 11.6$ according to Broser and Fleischhauer ${ }^{22}$ was used. The precise value of $\alpha_{\text {tr }}$ was checked spectrophotometrically for each solvent and at each temperature where kinetic runs were performed.

With the buffer concentrations used, the protolytic equilibria are expected to be established within microseconds, whereas the highest value of $k_{\text {eff }}$ measured was about $4 \times 10^{4} \mathrm{~s}^{-1}$. So the validity of eq 1 can be safely assumed.

The kinetics of thermal cis $\rightarrow$ trans isomerization was investigated at seven temperatures ranging between 15 and $55^{\circ} \mathrm{C}$. The reaction rate corresponds to a first-order rate law. From the temperature dependence of the rate constant the Arrhenius and Eyring activation parameters have been obtained.

In concluding the Experimental Section we point out that some kinetic data for thermal cis-trans isomerization of $\mathrm{M}$, reported a few years ago by Abdel-Mottaleb et al., ${ }^{23}$ were based on experiments where the role of $\alpha$ was not adequately taken into account. Therefore the rate parameters obtained in that work have no well-defined relation to the cis-trans isomerization process and should be disregarded.

\section{Results and Discussion}

The absorption spectra and the solvent dependence of the first absorption band are very similar for the merocyanines $M$ and $\mathrm{C}_{16} \mathrm{M}$. The spectra of the amphiphilic derivative are depicted in Figure 1 to demonstrate the general character of the spectral change. The $\epsilon_{\max }$ and $\lambda_{\max }$ values of both dyes are listed in Table I. Here are also given the Eyring activation parameters and the rate constants at $25^{\circ} \mathrm{C}$ of the thermal cis $\rightarrow$ trans isomerization process.

The results for $\mathrm{M}$ in water have been adopted from our previous work. ${ }^{18}$ The long-chain merocyanine $\mathrm{C}_{16} \mathrm{M}$ is actually insoluble in water, at least in a monomolecularly dispersed form. Experiments where methanolic stock solutions have been diluted with more than $50 \%$ of water show a drastic change of the absorption spectrum (a strong hypsochromic shift of $\lambda_{\max }$ and a drastic de-

(22) Broser, W.; Fleischhauer, H. Z. Naturforsch., B: Anorg. Chem., Org. Chem. 1970, 25, 1389.

(23) Abdel-Mottleb, M. S. A.; Abdel-Kader, M. H.; Lux, F. Z. Chem. (Leipzig) 1982, 22, 451. 
TABLE I: Spectroscopic ${ }^{a}$ and Kinetic Data for Thermal Cis-Trans Isomerization of Merocyanines $M$ and $\mathrm{C}_{16} \mathrm{M}^{b}$

\begin{tabular}{|c|c|c|c|c|c|c|}
\hline solvent & $\lambda_{\max }{ }^{a}$ & $\epsilon_{\max }{ }^{a}$ & $k_{\mathrm{ct}}{ }^{c}$ & $\Delta G_{\mathrm{ct}}^{*}{ }^{d, e}$ & $\Delta H_{\mathrm{ct}}^{d^{d, e}}$ & $\Delta S_{\mathrm{ct}}^{*}{ }^{e f}$ \\
\hline waterg & 442 & 39.0 & $7.4 \times 10^{-5}$ & 23.1 & 28.0 & 16.5 \\
\hline \multirow[t]{2}{*}{ ethylene glycol } & 474 & 46.3 & $3.4 \times 10^{-3}$ & 20.3 & 20.8 & 0.1 \\
\hline & $(480)$ & $(40.4)$ & $\left(6.0 \times 10^{-3}\right)$ & $(20.5)$ & (17.3) & $(-10.9)$ \\
\hline methanol & $\begin{array}{c}484 \\
(487)\end{array}$ & $\begin{array}{c}50.2 \\
(47.4)\end{array}$ & $\begin{array}{c}4.5 \times 10^{-2} \\
\left(7.7 \times 10^{-2}\right)\end{array}$ & $\begin{array}{c}19.3 \\
(19.0)\end{array}$ & $\begin{array}{c}20.6 \\
(20.8)\end{array}$ & $\begin{array}{c}4.2 \\
(6.2)\end{array}$ \\
\hline \multirow[t]{2}{*}{ 2-propanol } & 544 & 67.0 & 8.0 & 16.2 & 18.6 & 7.9 \\
\hline & (549) & (65.6) & $\left(1.1 \times 10^{1}\right)$ & $(16.0)$ & (19.3) & (10.9) \\
\hline \multirow[t]{2}{*}{ acetonitrile } & 547 & 73.2 & $5.3 \times 10^{1}$ & 15.1 & 19.0 & 13.2 \\
\hline & $(550)$ & $(68.0)$ & $\left(1.3 \times 10^{2}\right)$ & $(14.5)$ & $(21.8)$ & $(24.2)$ \\
\hline \multirow[t]{2}{*}{ dimethyl sulfoxide } & 573 & 99.4 & $1.2 \times 10^{-2}$ & 14.6 & 12.0 & -8.8 \\
\hline & $(577)$ & $(101.0)$ & $\left(1.9 \times 10^{-2}\right)$ & $(14.3)$ & $(11.0)$ & $(-11.1)$ \\
\hline \multirow[t]{2}{*}{$N, N$-dimethylformamide } & 580 & 99.5 & $5.7 \times 10^{2}$ & 13.7 & 14.7 & 3.2 \\
\hline & $(585)$ & $(93.2)$ & $\left(7.9 \times 10^{2}\right)$ & $(13.1)$ & (14.4) & $(2.4)$ \\
\hline \multirow[t]{2}{*}{ acetone } & 585 & 107.0 & $1.6 \times 10^{3}$ & 13.1 & 11.7 & -4.6 \\
\hline & (588) & $(114.0)$ & $\left(3.3 \times 10^{3}\right)$ & $(12.7)$ & $(13.3)$ & $(2.1)$ \\
\hline
\end{tabular}

${ }^{a} \lambda_{\max }(\mathrm{nm})$ and $\epsilon_{\max }\left(10^{3} \mathrm{M}^{-1} \mathrm{~cm}^{-1}\right)$ of the first absorption band of the basic trans isomer. ${ }^{b}$ Values for $\mathrm{C}_{16} \mathrm{M}$ are given in parentheses. ${ }^{\mathrm{c}} \mathrm{s}^{-1}$, value actually determined at $25^{\circ} \mathrm{C}$. ${ }^{d}$ Kilocalories. ${ }^{e}$ Calculated for $25^{\circ} \mathrm{C}$ from Arrhenius parameters. $/{ }^{\mathrm{cal}} /(\mathrm{mol} \mathrm{K})$. ${ }^{8} \mathrm{Data}$ from ref 18 .

crease in $\epsilon_{\max }$, along with a completely different band shape; similar spectra have been described by Donchi et al. ${ }^{21}$ ), which may be an indication of micellar aggregation.

Solvatochromism. An extensive investigation of solvatochromism of $\mathbf{M}$ has been recently reported by Jacques. ${ }^{12}$ Our results for $\lambda_{\max }$ of $\mathbf{M}$ are in very good agreement with his, except for acetonitrile, where our value of $547 \mathrm{~nm}$ is markedly shifted with respect to the value of $571 \mathrm{~nm}$ reported in his work. This discrepancy may be explained by the high sensitivity toward small amounts of protic solvents, reported for $\lambda_{\max }$ of $M$ in very dry acetonitrile. ${ }^{12}$ In our case the necessity of using buffered systems precluded the usage of solvents completely free of traces of protic cosolvents. Since we wanted to correlate spectral and kinetic behavior, we used the same quality of solvents for both types of investigations.

For $\mathrm{C}_{16} \mathrm{M}$ we found that the $\lambda_{\max }$ values are consistently at 3-6 $\mathrm{nm}$ longer wavelength than for $\mathrm{M}$. With respect to the large solvatochromic shifts this is only a minor change, indicating that the spectroscopic behavior of the chromophore is very slightly dependent on the alkyl substitutent. However, the shift is systematic and indicates that the effect of the long chain is to render the environment of the chromophore a little less polar than it is for $\mathrm{M}$ in the same solvent. A purely electronic effect of the alkyl substituent on the ring nitrogen (enhanced inductive shift) would be expected to cause a spectral shift in the same direction as if protonating the phenolic oxygen, which is known to cause a hypsochromic shift. This electronic effect must be obviously overcompensated for by the general environmental polarity effect exhibited by the long alkyl substituent. As is shown in Figure 2 the first electronic transition energy $\Delta E_{\max }$ of $\mathrm{M}$ and $\mathrm{C}_{16} \mathrm{M}$ correlates very well with the empirical solvent parameter $E_{\mathrm{T}}$. This is not too surprising since Dimroth's reference compound, a pyridinium- $N$-phenolate betaine, ${ }^{24}$ is closely related in electronic structure to the merocyanines investigated here. Taking the data for $\mathrm{M}$ and $\mathrm{C}_{16} \mathrm{M}$ together, we obtain correlation 3

$$
\Delta E_{\max }=0.803 E_{\mathrm{T}}+14.15 \mathrm{kcal} \quad(r=0.991)
$$

with similar coefficients as reported by Jacques ${ }^{12}$ for $\mathrm{M}$ only:

$$
\Delta E_{\max }=0.799 E_{\mathrm{T}}+14.56 \mathrm{kcal} \quad(r=0.989)
$$

In Figure 2 we have also plotted the solvatochromic data reported for DENAB. ${ }^{19}$ With these the correlation has a slope opposite to that of $M$. Furthermore, the correlation shows much more scatter. On the other hand, it has been reported by Schanze, Mattox, and Whitten ${ }^{19}$ (SMW) that when Taft's $\pi^{*}$ polarity scale is used, the DENAB data correlate fairly well:

$\Delta E_{\max }(\mathrm{kcal})=-6.88 \pi^{*}+63.00 \quad(r=0.986)$

(4-SMW)

(24) Dimroth, K.; Reichardt, C.; Siepmann, T.; Bohlmann, F. Liebigs Ann Chem. 1963, 661, 1. Dimroth, K.; Reichardt, C. Ibid. 1969, 727, 93 . Reichardt, C. Ibid. 1971, 752, 64. Maksimovic, Z. B.; Reichardt, C.; Spiric, A. Z. Anal. Chem. 1974, 270, 100.



Figure 2. Plot of transition energy $\Delta E_{\max }$ versus Dimroth's solvent parameter $E_{\mathrm{T}}: \bullet, \mathrm{M} ; \mathrm{O}, \mathrm{C}_{16} \mathrm{M} ; \square, \mathrm{DENAB}$ in aprotic solvents; $\times, \mathrm{DENAB}$ in protic solvents (DENAB data after Schanze et al. ${ }^{19}$ ).

This is not so in the case of $\mathrm{M}$ and $\mathrm{C}_{16} \mathrm{M}$. Analyzing the $\Delta E_{\max }$ data for $\mathrm{M}$, Jacques showed that these are similarly sensitive to $\pi^{*}$ and the hydrogen-bond-donor ability parameter $\alpha$ :

$\Delta E_{\max }(\mathrm{kcal})=9.69 \pi^{*}+11.13 \alpha+41.01 \quad(r=0.992)$

From our data, including those for both $\mathrm{M}$ and $\mathrm{C}_{16} \mathrm{M}$, we obtain the following correlation:

$\Delta E_{\max }(\mathrm{kcal})=9.78 \pi^{*}+11.45 \alpha+40.69 \quad(r=0.973)$

Thus we may note here that with respect to their solvatochromism, $\mathrm{M}$ and $\mathrm{C}_{16} \mathrm{M}$ differ from DENAB not only in the sign of the effect but also in their specific sensitivity to solvent hy. drogen-bond-donor effects.

Looking at the band shapes, yet another feature of the merocyanine solvatochromism is borne out. In the series of solvents investigated there is a montonic increase of $\epsilon_{\max }$ with decreasing solvent polarity parameter. But in the same series the width of the absorption bands decreases in such a way that the oscillator strength, which has been found to vary in the range between 0.9 and 1.1 , is almost constant. Such a relation has been suspected by Jacques ${ }^{12}$ from a comparison of two solvents, namely, $\mathrm{CHCl}_{3}$ and $\mathrm{CH}_{3} \mathrm{OH}$. We shall provide an explanation of this regularity below.

Solvatokinetic Behavior. As is borne out by the data in Table I the rate constant of cis $\rightarrow$ trans isomerization at $25^{\circ} \mathrm{C}$ is increased by about 7 orders of magnitude when going from water to acetone. This behavior nicely parallels the polarity series of the $\Delta E_{\max }$ data. The results for $\mathrm{M}$ and $\mathrm{C}_{16} \mathrm{M}$ are very similar, yet with the notable difference that in each solvent the rate constant for $\mathrm{C}_{16} \mathrm{M}$ is a factor of about 2 larger than for $\mathrm{M}$. We tend to assume that this difference is in itself a microenvironmental 


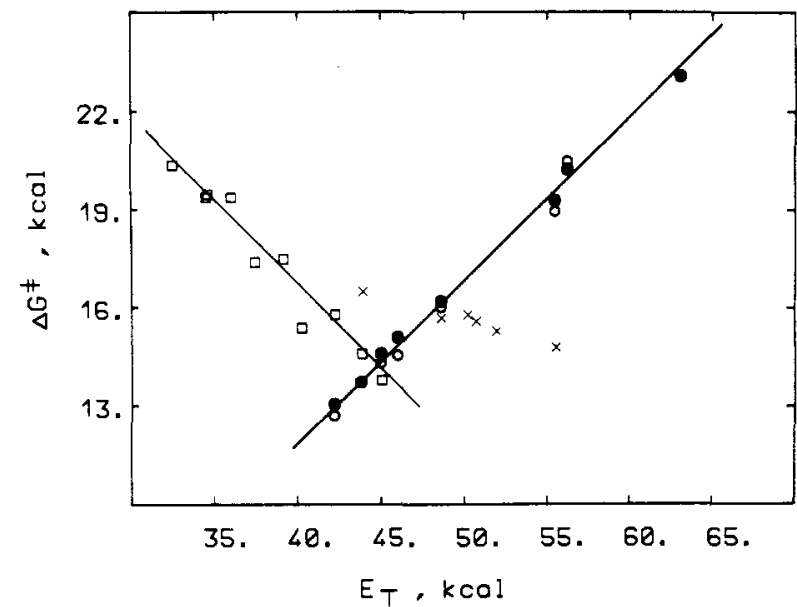

Figure 3. Plot of activation free enthalpy $\Delta G^{*}$ of thermal cis-trans isomerization versus Dimroth's solvent parameter $E_{\mathrm{T}}: \bullet, \mathrm{M} ; 0, \mathrm{C}_{16} \mathrm{M}$; $\square$, DENAB in aprotic solvents; $X$, DENAB in protic solvents (DENAB data after Schanze et al. ${ }^{19}$ ).

polarity effect and systematically reflects the small difference of $\Delta E_{\max }$ for both dyes, which we have interpreted as an indication of a somewhat lower polarity in the environment of the $C_{16} \mathrm{M}$ chromophore due to the long alkyl-chain substituent.

The solvatokinetic behavior of $M$ and $\mathrm{C}_{16} \mathrm{M}$ is almost clearly represented in Figure 3, where we have plotted the activation free enthalpy $\Delta G^{*}$ ct as a function of solvent polarity parameter $E_{\mathrm{T}}$. A good linear correlation is obtained. Including both the data for $\mathrm{M}$ and $\mathrm{C}_{16} \mathrm{M}$, the correlation parameters are

$$
\Delta G^{*}{ }_{\mathrm{ct}}=0.491 E_{\mathrm{T}}-7.760 \mathrm{kcal} \quad(r=0.996)
$$

Again it is interesting to compare the merocyanine case with the DENAB case, which is also depicted in Figure 3. With DENAB in aprotic solvents there is also a good correlation with $E_{\mathrm{T}}$, the slope corresponding approximately to the negative value of the correlation for M. The DENAB data for protic solvents (a series of alcohols) strongly depart from the main correlation line and seem to be distributed along another straight line. This has been noted previously by Whitten and co-workers ${ }^{25}$ for a plot using Kosowers $Z$ instead of $E_{\mathrm{T}}$. Since, however, $Z$ is linearly correlated with $E_{\mathrm{T}}{ }^{26}$ the conclusions are equivalent.

As discussed above, a linear correlation with $E_{\mathrm{T}}$ indicates the importance of hydrogen-bond-donor properties of the solvents if protic solvents are included in the correlation. Correspondingly the correlation with Taft's $\pi^{*}$ polarity parameter alone is very poor and needs improvement by a two-parameter correlation, including also the $\alpha$ parameter. In the case of $\Delta G_{\mathrm{ct}}$ we obtain

$$
\Delta G^{*}{ }_{\mathrm{ct}}(\mathrm{kcal})=6.34 \pi^{*}+7.11 \alpha+8.16 \quad(r=0.991)
$$

whereas Whitten's results for DENAB were

$$
\Delta G^{*}{ }_{\mathrm{ct}}(\mathrm{kcal})=-6.33 \pi^{*}+20.77 \quad(r=0.935)
$$

for aprotic solvents and

$$
\Delta G_{\mathrm{ct}}(\mathrm{kcal})=-6.33 \pi^{*}-2.38 \alpha+20.80 \quad(r=0.991)
$$

for protic solvents. Thus from a comparison of DENAB with $M$ and $\mathrm{C}_{16} \mathrm{M}$ in Figure 3 and eq 6 we see, that not only the solvatochromic but also the solvatokinetic behaviors of these compounds are of different sensitivity with respect to the specific solvent-solute interaction of hydrogen-bond formation.

As compared to $\Delta G^{*}$ the activation parameters $\Delta H^{*}$ ct and $\Delta \boldsymbol{S}^{*}{ }_{\mathrm{ct}}$ for $\mathrm{M}$ and $\mathrm{C}_{16} \mathrm{M}$ show considerable scatter in a polarity correlation. The correlation parameters for $\Delta H^{*}$ ct are

(25) Schanze, K. S.; Mattox, T. F.; Whitten, D. G. J. Am. Chem. Soc. 1982, 104, 1733 .

(26) Reichardt, C. Solvent Effects in Organic Chemistry; Verlag Chemie: Weinheim, 1978 .

$$
\Delta H_{\mathrm{ct}}^{*}=0.58 E_{\mathrm{T}}-11.00 \mathrm{kcal} \quad(r=0.80)
$$

which indicates that, on the average, the solvent-polarity dependence of $\Delta H^{*}$ ct is similar to that of $\Delta G^{*}$ ct.

When discussing the reasons for the observed solvatokinetic behavior of $\mathrm{M}$ and $\mathrm{C}_{16} \mathrm{M}$ in more detail, we may certainly rely on the assumption that in these compounds the mechanism of thermal cis-trans isomerization is an adiabatic rotation around the central $\mathrm{C}-\mathrm{C}$ bond. No other path connecting cis and trans form of our compounds, e.g., a motion analogous to the planar $\mathrm{N}$-inversional mechanism in azo compounds, suggests itself in our stilbene-type compounds. Furthermore, a diabatic change from the lowest singlet potential surface to the lowest triplet surface as discussed for some styryl pyridines ${ }^{27}$ seems to be highly improbable in view of the high-frequency factors which we found to be larger than $10^{11} \mathrm{~s}^{-1}$ in all solvents investigated.

Some quantum chemical data obtained by Tavan and Schulten ${ }^{16}$ on the change of electronic structure during cis-trans isomerization of $\mathrm{M}$ and $\mathrm{MH}^{+}$are of special interest in relation to our work. The protonated form $\mathrm{MH}^{+}$, while corresponding to b-type resonance structure in its planar trans conformation and its approximately planar cis conformation, obtains a good deal of q-type resonance character in the $90^{\circ}$ rotated conformation (henceforth denoted as $\mathrm{M}_{*}$ ), which is borne out in a pronounced charge migration from the phenyl group to the pyridyl group. This is just what would be expected by chemical intuition.

On the other hand, the unprotonated $\mathrm{M}$, which corresponds to a q-type structure in its planar trans or its approximately planar cis conformation, does not show such a change during isomerization. This is also in accord with chemical common sense, since the q-type structure with its low double-bond character of the central $\mathrm{C}-\mathrm{C}$ bridge is the most favorable one for the perpendicular conformation, anyway. Actually, according to the calculation of Tavan and Schulten, who considered isolated molecules devoid of any solvation, the $\mathrm{M}_{\mathrm{c}}$ conformation is even higher in energy than the perpendicular one, meaning that in the gas phase $\mathbf{M}_{c}$ would be thermodynamically unstable, i.e., the observability of $\mathbf{M}_{\mathrm{c}}$ in itself seems to be a solvent effect. An extension of the quantum chemical calculations to account for solvation would be highly desirable. So far, solvent effects have been taken into account only in a calculation confined to the planar $\mathbf{M}_{\mathrm{tr}}$ structure. ${ }^{15}$

As long as more detailed quantum chemical information is not available, one may assume as a rough approximation that the role of solvent polarity, no matter if it is actually due to hydrogen-bond formation, could be envisaged as being equivalent to exhibiting partial protonations ${ }^{14}$ at the oxygen atom, thus inducing a weaker or stronger contribution of resonance structure $b$ in the planar conformations, generating a corresponding molecular dipole moment and causing an energetic stabilization with respect to the nonpolar, q-type structured $M_{*}$ form.

Such a concept suggests that solvent-polarity-dependent changes of $\Delta G^{\ddagger}$ at are largely due to changes in the free energy of solvation $\Delta G_{\text {solv }}\left(\mathrm{M}_{\mathrm{c}}\right)$, viz.

$$
\Delta \Delta G_{\text {ct }}^{*} \approx \Delta \Delta G_{\text {solv }}\left(\mathrm{M}_{\mathrm{c}}\right)
$$

In the activated-state $M_{*}$, the solvation must be assumed to be in equilibrium with the actual charge distribution of the molecule. If this charge distribution closely corresponds to the q-type resonance structure, then the solvent will be only weakly polarized. On the other hand, if this unpolarized solvent configuration would be held fixed, a planarization of $M_{*}$ toward the $\mathrm{M}_{\mathrm{c}}$ conformation would be probably only weakly, if at all, exergonic, as can be concluded from Tavan and Schulten's results. Thus it follows that a descent from $M_{*}$ to $M_{c}$ or, vice versa, the activation from $M_{c}$ to $M_{*}$ will occur along a reaction coordinate corresponding to a highly cooperative motion of $\mathrm{C}-\mathrm{C}$ bond twist and orientational polarization of the solvent. These cooperative changes may be instigated by independent fluctuations of either the internal rotational vibration of $\mathrm{M}$ around the central $\mathrm{C}-\mathrm{C}$ bond or the orientational polarization of the solvent shell.

(27) Bortolus, P.; Cauzzo, G. Trans. Faraday Soc. 1970, 66, 1161 


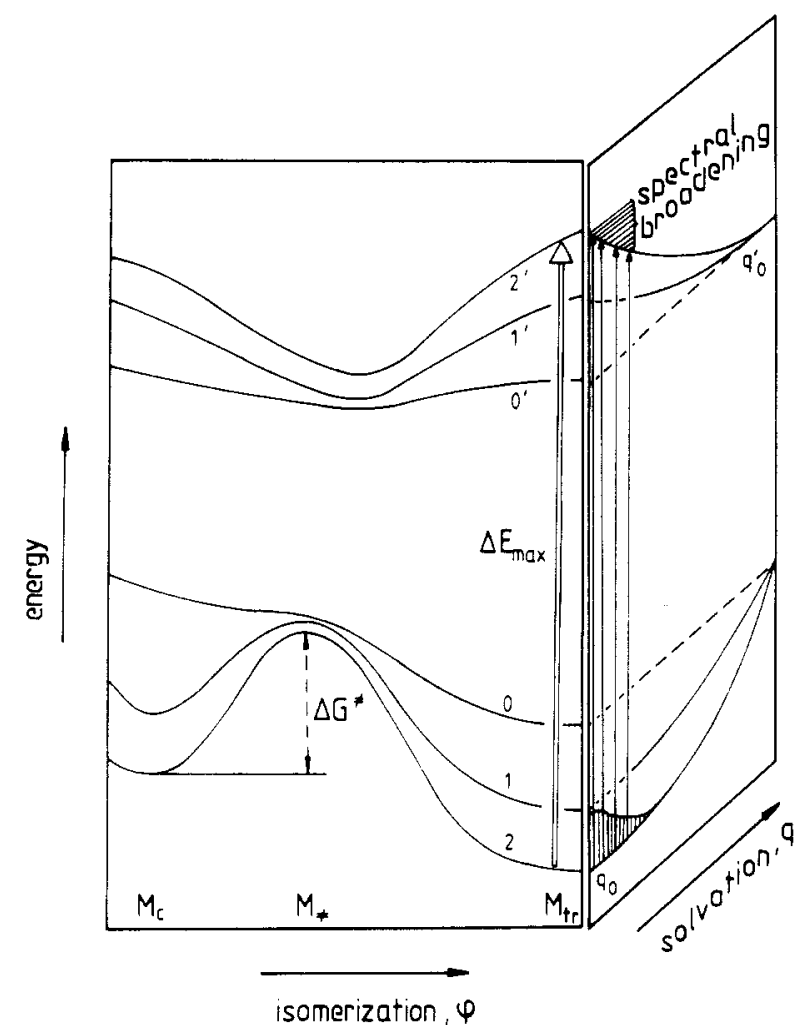

Figure 4. Schematic energy surfaces of $M$ versus cis-trans isomerization coordinate $\varphi$ and solvation coordinate $q$. The numbers 0,1 , and 2 in $S_{0}$ and $0^{\prime}, 1^{\prime}$, and $2^{\prime}$ in $S_{1}$ denote potential curves for increasing polarity of solvent.

In the case of DENAB these arguments apply similarly, with, however, the correspondence that here the activated state is the strongly polar one, and eq 8 would take the form

$$
\Delta \Delta G^{*}{ }_{\mathrm{ct}}(\mathrm{DENAB}) \approx \Delta \Delta G_{\mathrm{solv}}\left(\mathrm{DENAB}_{*}\right)
$$

Such a relation was implicitely assumed by Schanze et al. ${ }^{19}$ to estimate the change of dipole moment between the almost planar cis and the activated perpendicular conformation.

Correlation of Adiabatic and Diabatic Transition Energies. Traditionally, ${ }^{3,8}$ strongly negative solvatochromy has been interpreted as evidence that there is a strong decrease of the dipole moment on electronic excitation. Furthermore, our previous experimental investigations ${ }^{18}$ have shown that $\mathbf{M}_{c}$ photoisomerizes very efficiently, indicating that in the photoreactive state, which is thought to be the $S_{1}$ state, the double-bond character of the central $\mathrm{C}-\mathrm{C}$ bridge must be very low. Thus, with respect to charge distribution and central $\mathrm{C}-\mathrm{C}$ bond order the electronic structure of $M$ in $S_{1}$ ought to be very similar to that of the ground-state $M_{*}$ conformation. In particular this similarity is borne out in the linear correlation of both $\Delta G^{*}$ a and $\Delta E_{\max }$ with $E_{\mathrm{T}}$, implying, of course, that there is a direct linear correlation between $\Delta G^{*}{ }_{\mathrm{ct}}$ and $\Delta E_{\max }$. The correlation equation is

$$
\Delta G^{*}{ }_{\mathrm{ct}}=0.604 \Delta E_{\max }-15.98 \mathrm{kcal} \quad(r=0.989)
$$

Altogether our findings are suggestive of an explanation that may be illustrated by means of the schematic diagram depicted in Figure 4. Here the first horizontal axis corresponds to the rotational isomerization coordinate $\varphi$, whereby it is understood that along $\varphi$ the solvent arrangement is always in equilibrium with the actual intramolecular charge distribution in $M$. Let curve
1 represent the situation for a solvent of medium polarity; then changing to a more polar solvent (curve 2 ) will lower the potential minima at the close-to-planar conformations $\mathrm{M}_{\mathrm{c}}$ and $\mathrm{M}_{\mathrm{tr}}$, whereas the energy of the weakly polar transition-state conformation $M_{\neq}$ should be only weakly solvent dependent.

The potential curves $1^{\prime}$ and $2^{\prime}$ in the excited $S_{1}$ state belong to solvation states corresponding to vertical Franck-Condon (FC) transitions from the corresponding position of the $S_{0}$ isomerization potential curves 1 and 2, respectively. Since the electronic charge distribution changes on excitation, the FC solvent configuration is not in equilibrium with the new charge distribution. Thus, even though the $S_{1}$ state may have a small dipole moment, its FC energy will strongly depend on solvent polarity. In this way the diagram represents our finding that $\Delta \Delta E_{\max }>\Delta \Delta G^{*}{ }_{\mathrm{c}}$. On the other hand, the experimentally appearing proportionality of these quantities implies that the stabilization of $\mathrm{M}_{\mathrm{c}}$ (and $\mathrm{M}_{\mathrm{tr}}$ ) by increasing the solvent polarity must be proportional to the destabilization of the $\mathrm{S}_{1} \mathrm{FC}$ state.

Finally, to explain the solvent-polarity dependence of the absorption band width and its relation to the solvent dependence of $\Delta E_{\max }$, we have extended the potential diagram at a fixed position of the isomerization coordinate corresponding to $\mathrm{M}_{\mathrm{tr}}$, by adding the dimension of a representative solvation coordinate $q$. This coordinate can be defined as corresponding to a solvent configuration that would be established in equilibrium with a molecule $\left(\mathrm{M}_{\mathrm{tr}}\right)^{\prime}$ with a virtual dipole moment of $q .^{28}$ (One should note that $q$ is not constant along the reaction coordinate $\varphi$ as defined here.)

In the electronic ground state there will be a thermal distribution along the coordinate $q$, centered at $q_{0}$, the position of the potential minimum. In the excited state there is a solvent-polarity-dependent slope toward the excited-state equilibrium solvation $q_{0}^{\prime}$. It follows from the FC principle that the $q$ distribution in $\mathrm{S}_{0}$ is transferred to a $\Delta E$ distribution of the corresponding $F C$ transition, which is broader the steeper the slope in the excited-state potential curve. Thus, according to our model, the band width will increase in parallel with the transition energy as the polarity of the solvent increases.

\section{Conclusion}

Our experimental results demonstrate that for the merocyanine $M$ and its amphiphile derivative $C_{16} M$ the energy and band with of the first electronic transition and the activation free enthalpy $\Delta G^{*}$ th of thermal cis-trans isomerization are strongly solventpolarity dependent and correlate closely with each other. All these effects have been suggested to be related to the strong coupling between solvent polarization modes and conformationally induced electronic changes of the molecule going along with major charge redistribution. A detailed quantum chemical study of the relevant potential surfaces in the ground and excited states, including a suitable solvent polarization mode, would be highly desirable to achieve a better understanding of this intriguing situation.

Acknowledgment. This work was supported by the Scientific Channel Program of the Egyptean and German Federal Government. The cooperation of Prof. Dr. R. M. Issah and Prof. Dr. M. M. Ghoneim from Tanta University, Egypt, is gratefully acknowledged. Thanks are due to the Fonds der Chemischen Industrie for financial support.

Registry No. M, 23302-83-2; $\mathrm{C}_{16} \mathrm{M}$, 58346-32-0.

(28) Such a definition has been introduced by Marcus ${ }^{29}$ in relation to electron-transfer theory.

(29) Marcus, R. A. J. Chem. Phys. 1956, 24, 966, 979. 